OPEN ACCESS

Edited by:

Wang Lin,

Zhejiang University, China

Reviewed by:

West Greenlee M. Heather, lowa State University, United States

Henri Leinonen

University of California, Irvine, United

States

*Correspondence: Zilong HaO, zhilong1983@126.com

Bo Wu,

dr.bowu@hotmail.com

tThese authors have contributed equally to this work and share first authorship

Received: 06 November 2020 Accepted: 02 February 2021

Published: 25 February 2021

Citation:

Kwapong WR, Yan Y, Hao Z and Wu B (2021) Reduced Superficial Capillary Density in Cerebral Infarction Is Inversely Correlated With the NIHSS Score.

Front. Aging Neurosci. 13:626334. doi: 10.3389/fnagi.2021.626334

\section{Reduced Superficial Capillary Density in Cerebral Infarction Is Inversely Correlated With the NIHSS Score}

\author{
William Robert Kwapong ${ }^{\dagger}$, Yuying Yan ${ }^{\dagger}$, Zilong Hao* and Bo Wu* \\ Department of Neurology, West China Hospital, Sichuan University, Chengdu, China
}

Purpose: The retina and the brain share similar neuronal and microvascular features, therein we aimed to assess the structural and microvascular changes in the macula and choriocapillaris (CC) in patients with cerebral infarction when compared with healthy controls using optical coherence tomography angiography (OCTA).

Methods: OCTA was used to image and measure the capillary density in the radial peripapillary capillaries (RPC), superficial capillary plexus (SCP), deep capillary plexus (DCP), choriocapillaris (CC), and mean area of the foveal avascular zone (FAZ) in all participants. Twenty-two cerebral infarction patients based on their magnetic resonance imaging (MRI) and 25 healthy controls were included in our study.

Results: Density of the RPC $(P<0.001)$, SCP $(P=0.001)$, DCP $(P<0.001)$ and CC $(P<0.001)$ were significantly reduced in cerebral infarction patients when compared with healthy controls, respectively. Retinal thickness measurements $(P<0.05)$ were significantly reduced in cerebral infarction patients when compared with healthy controls. The mean FAZ area was significantly larger $(P=0.012)$ in cerebral infarction patients when compared with healthy controls. National Institute of Health Stroke Scale (NIHSS) inversely correlated with SCP density in cerebral infarction patients (Rho $=-0.409$, $P=0.001)$. Receiver operating characteristics curve analysis showed that the blood flow of the choriocapillaris had the highest index [area under the receiver operating characteristic $(A \cup R O C)=0.964$ ] to discriminate cerebral infarction patients from the healthy controls.

Conclusions: Our study suggests that cerebral microcirculation dysfunction which occurs in cerebral infarction is mirrored in the macula and choroidal microcirculation. OCTA has the potential to non-invasively characterize the macula and choroidal changes in cerebral infarction in vivo.

Keywords: cerebral infarction, macula capillaries, choriocapillaris, optical coherence tomography angiography, foveal avascular zone

\section{INTRODUCTION}

Stroke has been reported to be one of the common causes of death worldwide and is the leading cause of death in China (Zhou et al., 2019). Zhou et al. (2019) reported that with the rapid socioeconomic transition in China, the prevalence of stroke and its risk factors is bound to increase. The pathological changes of stroke involve a decrease in blood flow which leads to insufficient 
oxygen and cell death in the brain and spinal cord. Unfortunately, the classical clinical manifestation of ischemic stroke, which comprises abrupt unresponsiveness in the face, arm, or leg, particularly on one side of the body, is only apparent after immense blood flow loss in the brain and cell death. Although the neurological discrepancies are typical examples of stroke sequelae, these discrepancies go together with stroke-induced visual dysfunction in over $60 \%$ of stroke cases (Rowe, 2017).

Cerebral infarction, a common incidental discovery on magnetic resonance imaging (MRI), has been reported to be linked to ischemic stroke and help foretell its development (Vermeer et al., 2007); it has also been suggested that cerebral infarction is associated with subtle neurological deficits (Vermeer et al., 2007). Nonetheless, the understanding of the pathophysiology of cerebral infarction remains inadequate. Although cerebral microcirculation dysfunction (mainly cerebral ischemia) has been suggested to play a crucial role in the disease pathogenesis of cerebral infarction, gaining more comprehension is challenging due to the scarcity of non-invasive tools that evaluate the cerebral microcirculation quantitatively and consistently.

The retina, an extension of the central nervous system, has been reported to share many neuronal and microvascular features with the brain because of their similar anatomy, physiology, and embryology (Erskine and Herrera, 2014); besides, accumulating reports (Ong et al., 2013, 2015) have suggested that retinal thickness and microvasculature reflect the microstructural and microcirculation of the brain. Recent reports have shown that the retina is a reliable route to access cerebral microcirculation because of their similarities (London et al., 2013). Previous reports (Cooper et al., 2006; Ikram et al., 2006; de Jong et al., 2008; Cheung et al., 2010) using retinal imaging tools such as the fundus camera have suggested that retinal vascular damage such as retinal hemorrhage and microaneurysms are associated with predictors of stroke, cerebral white matter lesions, and other cerebrovascular diseases. Moreover, retinal ischemia, ischemia which affects the retinal cell loss and damage of the optic nerve, has been suggested to co-exist with cerebral infarction and may be responsible for many of the visual impairments that occur in this disease (Osborne et al., 2004; Cooper et al., 2006). Nonetheless, these retinopathy manifestations have been reported to be somewhat late pointers of retinal damage and reflect the late phase of retinal microvascular and structural damage.

Optical coherence tomography angiography (OCTA) is an imaging tool that can non-invasively image the retinal and choroidal microcirculation in vivo; OCTA helps clinicians to visualize the retinal and choroidal capillary anastomoses at a high resolution. Advances in the imaging and quantification software of the OCTA have now enabled a more objective quantitative assessment of the capillary network in the retina and choroid, which reflect earlier and subtler changes in the retinal and choroidal microcirculation before the apparent retinal signs emerge.

In this study, we assessed whether OCTA technology has the potential to characterize the retinal structure and retinal and choroidal microvascular changes in patients with cerebral infarction.

\section{MATERIALS AND METHODS}

\section{Data Avaliability Statement}

The data that support the findings of this study are available on request from the corresponding author. The data are not publicly available due to privacy or ethical restrictions.

\section{Study Population}

Patients presenting to the Neurology Department of West China Hospital, China, were recruited from May 2020 to October 2020. Patients were included if they were Chinese, had cerebral infarction confirmed with magnetic resonance imaging (MRI), could sit adequately, and tolerate retinal imaging using the OCTA. Infarction lesions were confirmed on MR diffusionweighted imaging consistent with clinical symptoms, as shown in (Figure 1). MR and OCTA imaging were done within 7 days after admission to our hospital. National Institute of Health Stroke Scale (NIHSS) scores were documented. Inclusion criteria of our cerebral infarction patients were as follows: (1) age 20-70 years; and (2) had cerebral infarction which was identified within 1 week of onset. Eligible cerebral infarction cases were matched to controls of the same age group, sex, and race with no self-reported history of stroke or any neurological deficits. Written informed consent was obtained from each participant and approval of our project was obtained from the Ethics Committee of West China Hospital of Sichuan University.

The exclusion criteria of our participants were as follows: (1) diagnosed with diabetic retinopathy or other retinal diseases; (2) Glaucoma; (3) presence of metal fragments in the eyes, brain, or spinal cord; (4) significant media opacities that preclude imaging of the macula; (5) participants with uncertain diagnoses; and (6) participants with a pacemaker or other internal devices; Ultimately, 25 patients were recruited for the ischemic stroke group, and 25 age- and sex-matched controls were enrolled.

After collecting the demographics and clinical information of the participants, ophthalmological examinations such as visual acuity, fundus examination, intraocular pressure examination, and spectral-domain optical coherence tomography (SD-OCT) imaging were completed.

Visual acuity under illumination was completed for each eye using Snellen charts and later converted to the logarithm of the minimum angle of resolution ( $\log M A R)$.

\section{Spectral-Domain Optical Coherence Tomography Imaging Macula Structure}

The peripapillary retinal nerve fiber layer (pRNFL) thickness was obtained using the optic nerve head map protocol; the scanning range covered a circle of $3.45 \mathrm{~mm}$ in diameter centered on the optic disc. Ganglion cell complex (GCC) thickness was obtained using the GCC scanning protocol which was acquired through scans centered $1 \mathrm{~mm}$ temporal to the fovea 

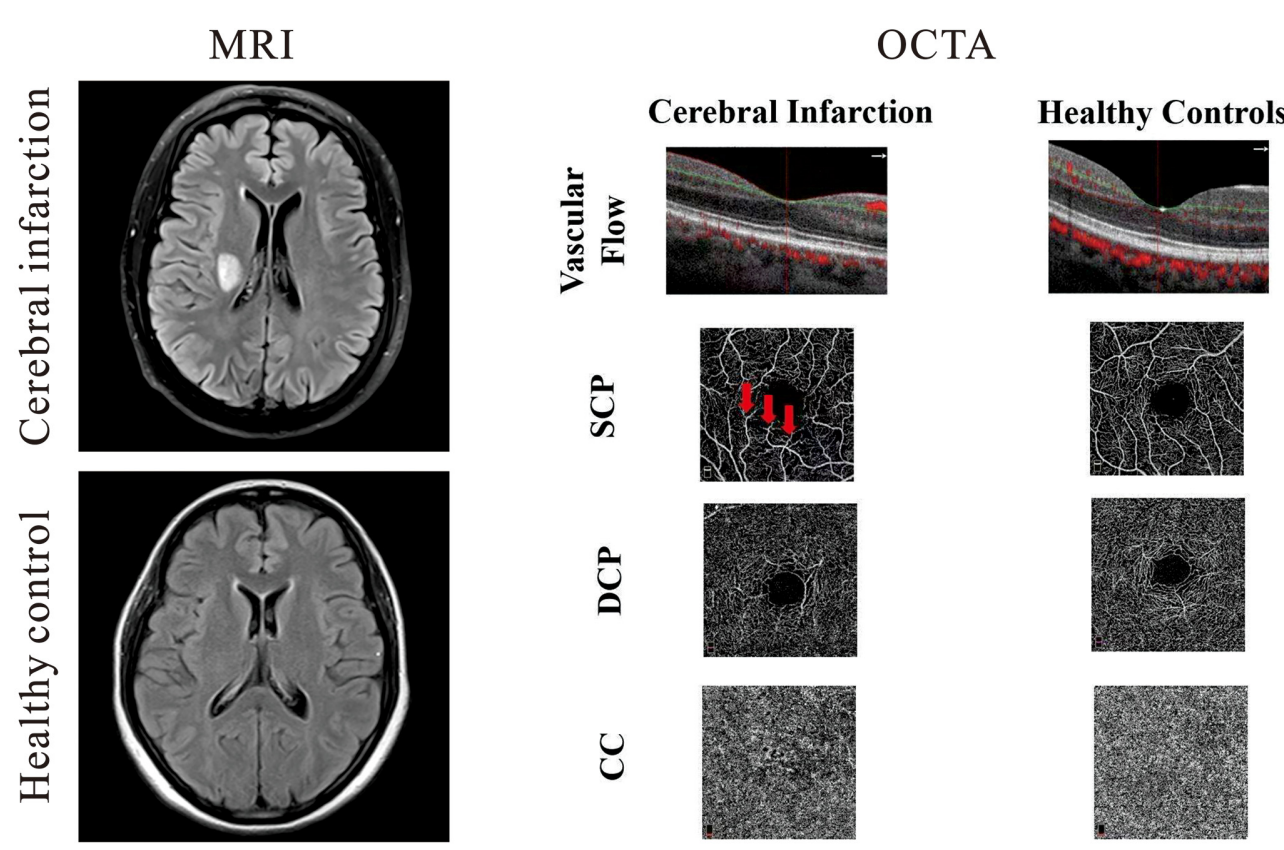

FIGURE 1 | Representative magnetic resonance imaging (MRI) and optical coherence tomography angiography (OCTA) images of cerebral infarction and healthy controls. On the left panel, the upper image shows a T2-weighted MRI of a patient with cerebral infarction beside the right lateral ventricle while the lower right image shows a T2-weighted image of healthy control. Cerebral infarction patients showed reduced vascular flow when compared with healthy controls; macula capillaries were more interrupted in cerebral infarction images when compared with healthy controls. Red arrows represent areas of capillary dropout (middle panel).

and covered a square grid $(7 \mathrm{~mm} \times 7 \mathrm{~mm})$ on the central macula. Imaging of the structure of the macula was done with the Avanti RTVue-XR (Optovue, Fremont, California, LA, USA, 2017 version).

\section{Capillaries of the Macula and Choroid}

Using the split-spectrum amplitude decorrelation algorithm, OCT angiography (OCTA) images were obtained with Avanti RTVue-XR. The built-in algorithm (version 2017.1.0.151) was equipped with a three-dimensional Projection Artifact Removal (3D PAR) to help reduce all projection artifacts in the deeper layers while keeping their outline and improving the foveal avascular zone (FAZ) area (Liu et al., 2018).

The radial peripapillary capillaries (RPC) network was obtained in scans within a $0.7 \mathrm{~mm}$ wide elliptical annular region extending outward from the optic disc boundary, and the vasculature within the internal limiting membrane and the retinal nerve fiber layer (RNFL) were analyzed using the built-in software.

The parafoveal capillary network was obtained through $3 \times 3 \mathrm{~mm}^{2}$ scans within the annular zone of $0.6 \mathrm{~mm}-2.5 \mathrm{~mm}$ diameter around the foveal center. The superficial capillary plexus (SCP) was $3 \mu \mathrm{m}$ below the internal limiting membrane to the outer boundary of the inner plexiform layer. The deep capillary plexus (DCP) was described as $15 \mu \mathrm{m}$ below the INL to $70 \mu \mathrm{m}$. The choriocapillaris (CC) was defined as the microvessels within the Bruch's membrane and the upper boundary of the stroma of the choroid.
The capillary density of the SCP, DCP, and CC was defined as the percentage area occupied by the microvasculature in the analyzed region $\left(3 \times 3 \mathrm{~mm}^{2}\right)$ and was generated in the whole scan area in all sections of the applied grid according to the Early Treatment Diabetic Retinopathy Study (ETDRS) as previously reported (Yang et al., 2019).

Images with signal quality less than 6 on a scale of 10 were excluded from our data analyses (Lim et al., 2018). Images with motion artifacts seen on the en face images such as irregular patterns of vessels or blurred segmentation of the microvascular plexuses were rejected. Images of good technical quality were included in our data analyses.

\section{Statistical Analyses}

Demographics and clinical information of our participants were described with frequencies (\%) and standard deviations as appropriate. Chi-square or independent sample $t$-tests were used to compare the clinical and demographic information between the two groups when appropriate. A generalized estimating equation (GEE) was used to compare the structure, macula microvasculature, and CC between cerebral infarction patients and healthy controls; after, these parameters were then assessed again using GEE while adjusting for inter-eye dependencies, signal quality, and risk factors (gender, hypertension, diabetes, and age). Intraocular pressure of each eye was included as a covariate when analyzing the macula structural thickness between the two groups. The area under the receiver operating characteristic (AUROC) was used to determine the diagnostic 
TABLE 1 | Demographics and clinical data.

\begin{tabular}{|c|c|c|c|}
\hline & $\begin{array}{l}\text { Cerebral } \\
\text { infarction }\end{array}$ & $\begin{array}{l}\text { Healthy } \\
\text { controls }\end{array}$ & $P$-value \\
\hline Number & 22 & 25 & \\
\hline Gender (F:M) & $2: 20$ & $3: 22$ & 0.891 \\
\hline Age (years) & $54.35(11.42)$ & $54.41(9.97)$ & 0.985 \\
\hline BMl & $24.23(2.54)$ & $23.95(2.16)$ & 0.793 \\
\hline Hypertension (number) & 9 & 3 & $<0.05$ \\
\hline Diabetes (number) & 5 & 1 & $<0.05$ \\
\hline Hyperlipidemia & 5 & 0 & $<0.05$ \\
\hline Smokers (number) & 14 & 9 & 0.083 \\
\hline Alcohol (Smokers) & 8 & 6 & 0.311 \\
\hline \multicolumn{4}{|l|}{ Location of infarction } \\
\hline Subcortical n (\%) & $15(68.2)$ & & \\
\hline Cortical and subcortical $n(\%)$ & $1(4.5)$ & & \\
\hline Brain stem & $6(27.3)$ & & \\
\hline NIHSS score & $2.53(2.32)$ & 0 & \\
\hline \multicolumn{4}{|l|}{ Ophthalmology exam } \\
\hline IOP (mmHg) & $15.28(2.48)$ & $14.96(2.32)$ & 0.812 \\
\hline $\mathrm{AL}(\mathrm{mm})$ & $23.16(1.19)$ & $23.45(0.78)$ & 0.958 \\
\hline Visual acuity, Snellen chart & $0.74(0.31)$ & $1.10(0.17)$ & $<0.001$ \\
\hline Visual acuity, LogMAR & $0.21(0.23)$ & $-0.05(0.07)$ & $<0.001$ \\
\hline
\end{tabular}

Data were expressed as mean (standard deviations) or percentages. Chi-square or independent sample $t$-tests were used in comparing characteristics between groups. Text in blue font indicates location of infarct.

accuracy of the analyzed parameters discriminating between cerebral infarction patients and healthy controls. An AUROC of 1.0 indicated perfect discrimination while 0.5 indicated accidental discrimination. $P$-values less than 0.05 were considered statistically significant. SPSS version 22 was used to perform the statistical analyses.

\section{RESULTS}

Twenty-five patients with cerebral infarction were enrolled in this study. Owing to the poor quality of OCTA images of both eyes (movement artifacts, segmentation errors, and signal quality less than 6), three cerebral infarction patients were excluded from our data analyses. Thus, 22 cerebral infarction patients and 25 healthy controls were included in our data analyses. Of our 22 cerebral infarction patients, 15 had subcortical infarction, one had cortical and subcortical infarction, and six had brain stem infarction as shown in (Table 1). There were no significant differences in age, gender, axial length, and IOP between the two groups. Significant differences $(P<0.05)$ were shown in the risk factors such as hypertension and diabetes between the two groups (Table 1). Cerebral infarction patients showed significantly reduced visual acuity when compared with healthy controls $(P<0.001)$.

Cross-sectional OCTA images of the macula with visible flow signal through the center of the fovea and in the circumferential portion of the macula were significantly different between the two groups; cerebral infarction patients showed significantly reduced vascular flow when compared with the healthy controls (Figure 1). On the en face angiograms, cerebral infarction patients showed narrower and interrupted capillaries when compared with healthy controls (Figure 1, middle column). Signal quality was significantly different $(P=0.03)$ between patients with cerebral infarction $(8.33 \pm 1.17)$ and healthy controls $(9.03 \pm 0.72)$.

\section{Macula Thickness Between Ischemic Stroke and Healthy Controls}

pRNFL thickness $(P=0.008)$, RNFL thickness $(P=0.02$, Table 2$)$ and GCC thickness $(P=0.018$, Table 2$)$ were significantly reduced in cerebral infarction patients when compared with healthy controls, respectively.

The mean FAZ area was significantly larger in cerebral infarction patients when compared with healthy controls $(P<0.001$, Table 2).

\section{The Capillary Density Between Ischemic Stroke and Healthy Controls}

RPC density $(P<0.001$, Table 2$)$, SCP density $(P=0.001$, Table 2$)$ and DCP density $(P<0.001$, Table 2$)$ were significantly reduced in cerebral infarction patients when compared with healthy controls, respectively. The density in the choriocapillaris was significantly reduced in cerebral infarction patients when compared with healthy controls $(P<0.001$, Table 2$)$.

Mean area of the FAZ in cerebral infarction patients inversely correlated with the DCP density (Rho $=-0.288$, $P=0.001)$ and blood flow in the choriocapillaris $($ Rho $=-0.264$, $P=0.002$ ), respectively.

NIHSS also inversely correlated with the SCP density in patients with cerebral infarction (Rho $=-0.409, P=0.001$ ).

AUROC analysis was used to reflect the diagnostic accuracy for each OCTA parameter to provide a distinction between cerebral infarction patients and healthy controls. The highest AUROC result was the choriocapillaris blood flow (0.964, 95\% $\mathrm{CI}=$ 0.889-0.994; Figure 2, Table 3).

\section{DISCUSSION}

In our observational cross-sectional study, we assessed the retinal, structural, and microvascular changes in patients with cerebral infarction. Our current study compared the sub-retinal thicknesses and microvascular densities in the two macula capillary plexuses (superficial and deep), capillaries around the optic nerve, and choriocapillaris. When compared with healthy controls, patients with cerebral infarction showed significantly thinner sub-retina thickness (pRNFL, RNFL, and GCC) and significantly reduced RPC, SCP, DCP, and CC densities. Our report adds to the notion that gradual modifications occur in the retinal structure and microvasculature and choriocapillaris in patients with cerebral infarction. Taken together with accumulating reports, our current study shows that changes in retinal structure and microvasculature and choriocapillaris using the OCTA may potentially be used in monitoring the retinal changes in cerebral infarction.

Our findings on the sub-retinal layer thinning ( $\mathrm{pRNFL}$, RNFL, GCC) in cerebral infarction patients when compared with healthy controls are congruent with previous reports on cerebral vascular diseases (Kim et al., 2011; Moss, 2015). The pRNFL, RNFL, and GCC contains axons, cell bodies, and dendrites, thus a significant thinning of these layers reflects a significant neuronal 
TABLE 2 | Comparison of the macula and choriocapillaris between cerebral infarction and healthy controls.

\begin{tabular}{|c|c|c|c|c|}
\hline & Cerebral infarction & Healthy controls & $P$-value & $P$-value \\
\hline RPC (\%) & $49.02(3.0)$ & $53.01(2.54)$ & $<0.001$ & $<0.001$ \\
\hline pRNFL ( $\mu \mathrm{m})$ & $112.41(11.89)$ & $120.71(8.68)$ & 0.003 & 0.008 \\
\hline RNFL ( $\mu \mathrm{m})$ & $104.02(8.63)$ & $108.94(7.65)$ & 0.025 & 0.020 \\
\hline $\operatorname{GCC}(\mu \mathrm{m})$ & $98.43(6.73)$ & $101.74(5.05)$ & 0.014 & 0.018 \\
\hline SCP (\%) & 45.93 (3.69) & $48.22(1.43)$ & 0.002 & 0.001 \\
\hline DCP (\%) & $48.50(3.48)$ & $52.54(2.27)$ & $<0.001$ & $<0.001$ \\
\hline $\mathrm{FAZ}\left(\mathrm{mm}^{2}\right)$ & $0.30(0.10)$ & $0.22(0.63)$ & $<0.001$ & $<0.001$ \\
\hline Choriocapillaris (\%) & $61.61(4.58)$ & $68.75(1.11)$ & $<0.001$ & $<0.001$ \\
\hline
\end{tabular}

Data were adjusted for intereye dependencies, hypertension, diabetes, age, gender, and signal quality. P-value: adjusted for signal quality. P-value (italics): adjusted for signal quality and risk factor. Text in blue font indicates the significance of OCT changes with (last column, $P$ value indented) and without (first $P$ value column, $P$ value not indented) adjusting for risk factors.

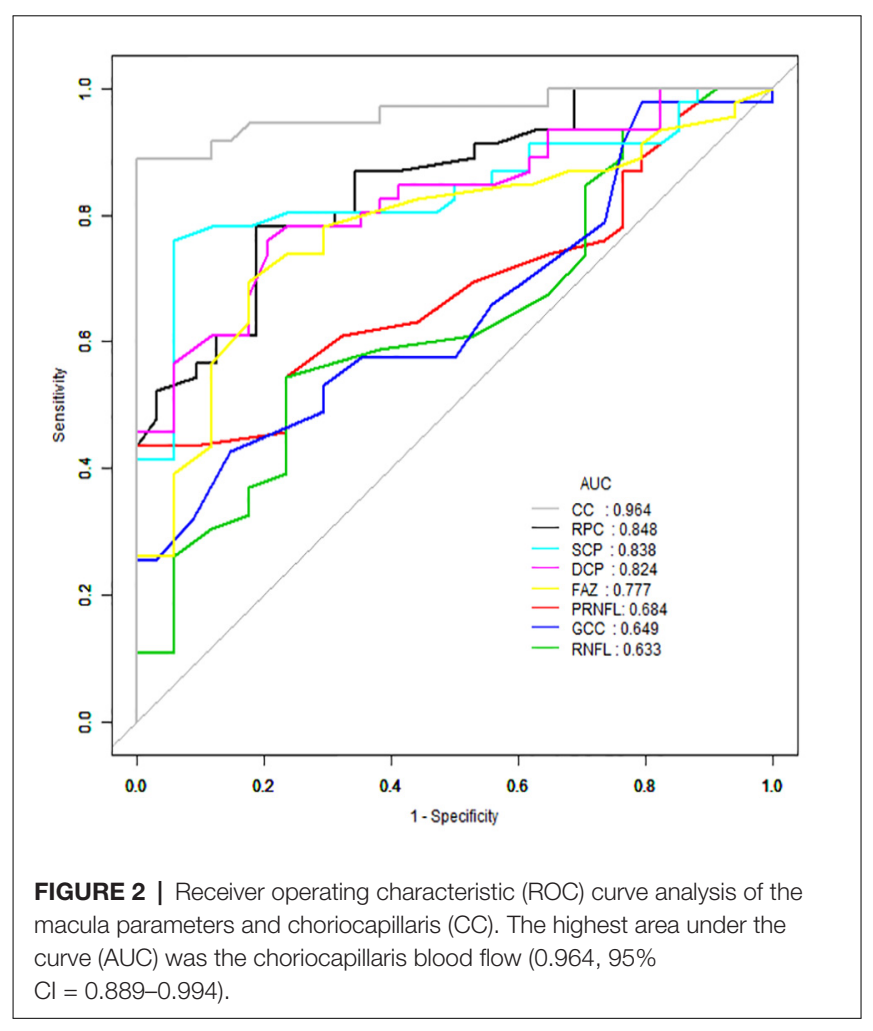

TABLE 3 | ROC curve analysis of the macula and choriocapillaris of cerebral infarction and healthy controls.

\begin{tabular}{lccc}
\hline & AUC & Sensitivity & Specificity \\
\hline RPC $(\%)$ & 0.848 & 78.26 & 81.25 \\
pRNFL $(\mu \mathrm{m})$ & 0.684 & 43.48 & 100 \\
RNFL $(\mu \mathrm{m})$ & 0.633 & 54.35 & 76.47 \\
GCC $(\mu \mathrm{m})$ & 0.649 & 42.55 & 85.29 \\
SCP $(\%)$ & 0.838 & 76.09 & 94.12 \\
DCP $(\%)$ & 0.824 & 76.09 & 79.41 \\
FAZ $\left(\mathrm{mm}^{2}\right)$ & 0.777 & 69.57 & 82.35 \\
Choriocapillaris $(\%)$ & 0.964 & 88.89 & 100 \\
\hline
\end{tabular}

and axonal loss which is congruent with previous cerebral reports (Brundel et al., 2012; Smith et al., 2012).

The growing number of reports (Mitchell et al., 2005; Baker et al., 2008; Wang et al., 2011; Kawasaki et al., 2012) providing evidence of significantly altered retinal microvascular structures in cerebral infarction indicates the importance of assessing the retinal microvasculature in vivo. This may shed light on the role of the microcirculatory networks and the development of the disease. Moreover, currently available and widely accepted imaging modalities such as magnetic resonance angiography are expensive, lack specificity, and have their contraindications. Therein, the use of retinal imaging may help in understanding the pathologic mechanism underlying the disease cascade and can be complementary to these neuroimaging tools. The OCTA is an imaging tool that helps in the acquisition of high-resolution in vivo crosssectional images and assessable measurements of the optic disc, macula, and choriocapillaris, which have been reported to be associated with the cerebral structures (microvasculature and microstructure). It has been suggested to be particularly useful in many neurodegenerative diseases and a useful tool in examining the course of development (Wylesgała, 2018; Pujari et al., 2020). The usefulness and potential of OCTA to determine capillary-level detail make it an important imaging modality that can provide in-depth information regarding impaired perfusion. It allows clinicians to assess the severity of ischemia (Pujari et al., 2020) with much more precision which may be beneficial in understanding the pathophysiology of cerebral infarction.

Our data observed a significant dropout of macula microvasculature (SCP and DCP) in cerebral infarction patients when compared with healthy controls. Previous cerebral imaging reports have suggested that vascular dysfunction leads to cerebral hypoperfusion during the development of ischemic cerebrovascular lesions (Kunz and Iadecola, 2009; Hu et al., 2017). Moreover, it has been suggested that before cerebral infarction, most patients may have had combinations of largevessel stenosis, small vessel disease, and a propensity to form either cardiac emboli or arterial thrombi with atherosclerotic lesions. This may then result in neural cell loss/death and a significant reduction in cerebral microcirculation dropout. With the association between cerebral microcirculation and retina microcirculation, the retinal microcirculation may be reflective of the pathophysiological cascade in cerebral infarction. Ideally, we found that the capillary damage in the deep plexus was more severe than in the superficial plexus. The deep capillary plexus which is located in the deepest part of the inner retina is reported to consist solely of capillaries, unlike the superficial plexus which consists of capillaries, arterioles, and venules. The capillaries in the DCP are thinner and have a smaller cross-sectional area 
making them sensitive to any disease that affects the retina. However, our study is observational and did not explore the contributory mechanisms.

Our report also showed that patients with cerebral infarction have a significantly reduced RPC density when compared with healthy controls. Our report is in agreement with a previous study that showed reduced vascular density around the optic nerve using fundus photography (Sprodhuber et al., 2018). Although vessels imaged and measured from fundus photography are considerably larger in diameter than those obtained from the OCTA (Forrester et al., 1996), our report echoes the aforementioned report.

A novel finding in our study was the significant density dropout in the choriocapillaris of cerebral infarction patients when compared with healthy controls. It has been suggested that the occurrence of cerebral infarction is associated with aging and atherosclerosis of the vessels (Lernfelt et al., 2002; Liu et al., 2017), which create an ischemic environment, therein affecting the structure and microvasculature of the choroid. Significant dropout in the choriocapillaris remained after adjusting for age and other risk factors suggesting that changes in the choriocapillaris due to the disease mechanism. Future studies will be needed to validate our hypothesis.

The FAZ is located in the inner retina (mostly in the deep capillary plexus region) and it is made up of photoreceptor cells and bound by interconnected capillary anastomoses (Tick et al., 2011; Bates et al., 2018). Our current study showed that the FAZ area was larger in cerebral infarction patients when compared with healthy participants and observed significant inverse associations between the FAZ area and DCP and CC, respectively. The FAZ enlargement in patients with cerebral infarction may be due to retinal degeneration as seen in our current report and previous reports. Another possible explanation for the FAZ enlargement may be the significant dropout in the microvascular as shown in our current report as well.

An inverse correlation between the NIHSS score and density in the SCP was another new finding in our report. NIHSS, an important indicator to assess the severity of a stroke, has been reported to reflect the severity of neurological impairment (Lyden, 2017). An inverse correlation between NIHSS score and the density in the SCP of patients with cerebral infarction indicates that the higher the NIHSS score, the lower the density in the SCP and vice versa. Although this is the first report to highlight the correlation between these two parameters, future studies are needed to validate our speculation.

The significant difference between healthy controls and cerebral infarction provides a potentially clinically useful screening indicator for detecting the presence of cerebral infarction. Despite a promising area under the curve (AUC) in the ROC analysis with a lower 95\% CI of greater than 0.5 , studies using a longitudinal approach may be needed to validate our findings. Nonetheless, our results suggest that measurement of the blood flow in the choriocapillaris using OCTA can be used to identify cerebral infarction patients and may constitute an early indicator to help screen for patients with cerebral infarction. Identifying the significant reduction of the choriocapillaris in cerebral infarction patients before the occurrence of the apparent retinopathy signs seen on fundus photography may help clinicians to apply earlier implementation of treatment and may be useful to predict the progression of the disease as well.

A major limitation in our current study is the small sample size and inclusion of only Chinese participants. Second, individuals were excluded with a known vascular disease from our study; therein, we could not determine whether these results were translatable to individuals with retinal and choriocapillaris changes due to other causes. Another limitation is the statistical difference in the clinical information such as the number of hypertensives and diabetics included in our data. The OCTA technique requires participants to focus and cooperate, which makes some of the images obtained unsuitable for analysis.

\section{CONCLUSIONS}

Notwithstanding our limitations, we showed that patients with cerebral infarction can be imaged and detected by the OCTA which is a quick, non-invasive, inexpensive tool based on the significant reduction of blood flow in the choriocapillaris. Our report showed that patients with cerebral infarction have significantly reduced macular microvascular and choriocapillaris densities when compared with healthy controls. We also showed that reduced SCP density is inversely correlated with NIHSS score in cerebral infarction patients. Our findings suggest that these microvascular changes in the retina and choriocapillaris may reflect the cerebral microcirculation in cerebral infarction and demonstrate the potential of OCTA for early screening of cerebral infarction patients.

\section{DATA AVAILABILITY STATEMENT}

The raw data supporting the conclusions of this article will be made available by the authors, without undue reservation.

\section{ETHICS STATEMENT}

The studies involving human participants were reviewed and approved by West China Hospital Ethics Committee. The patients/participants provided their written informed consent to participate in this study.

\section{AUTHOR CONTRIBUTIONS}

All authors listed have made a substantial, direct and intellectual contribution to the work, and approved it for publication.

\section{FUNDING}

This work was supported by National Key Development Plan for Precision Medicine Research (2017YFC0910004), National Natural Science Foundation of China (81671146 and 81870937), and the 1.3.5 project for disciplines of excellence, West China Hospital, Sichuan University (ZYG D18009). 


\section{REFERENCES}

Baker, M. L., Hand, P. J., Wang, J. J., and Wong, T. Y. (2008). Retinal signs and stroke: revisiting the link between the eye and brain. Stroke 39, 1371-1379. doi: 10.1161/STROKEAHA.107.496091

Bates, N. M., Tian, J., Smiddy, W. E., Lee, W. H., Somfai, G. M., Feuer, W. J., et al. (2018). Relationship between the morphology of the foveal avascular zone, retinal structure and macular circulation in patients with diabetes mellitus. Sci. Rep. 8:5355. doi: 10.1038/s41598-01823604-y

Brundel, M., de Bresser, J., van Dillen, J., Kappelle, L. J., and Biessels, G. J. (2012). Cerebral microinfarcts: a systematic review of neuropathological studies. J. Cereb. Blood Flow. Metab. 32, 425-436. doi: 10.1038/jcbfm. 2011.200

Cheung, N., Mosley, T., Islam, A., Islam, A., Kawasaki, R., Sharrett, R. A., et al. (2010) Retinal microvascular abnormalities and subclinical magnetic resonance imaging brain infarct: a prospective study. Brain 133, 1987-1993. doi: 10.1093/brain/awq127

Cooper, L. S., Wong, T. Y., Klein, R., Sharrett, A. R., Bryan, R. N., Hubbard, L. D., et al. (2006). Retinal microvascular abnormalities and MRI-defined subclinical cerebral infarction: the atherosclerosis risk in communities study. Stroke 37, 82-86. doi: 10.1161/01.STR.0000195134.04355.e5

de Jong, F. J., Vernooij, M. W., Ikram, M. K., Ikram, M. A., Hofman, A., Krestin, G. P., et al. (2008). Arteriolar oxygen saturation, cerebral blood flow and retinal vessel diameters. The rotterdam study. Ophthalmology 115, 887-892. doi: 10.1016/j.ophtha.2007.06.036

Erskine, L., and Herrera, E. (2014). Connecting the retina to the brain. ASN Neuro. 6:1759091414562107. doi: 10.1177/1759091414562107

Forrester, J., Dick, A., McMenamin, P., and Wr, L. (1996). The Eye: Basic Science in Practice, 2nd Edition Edinburgh: Saunders.

Hu, X., De Silva, T. M., Chen, J., and Faraci, F. M. (2017). Cerebral vascular disease and neurovascular injury in ischemic stroke. Circ. Res. 120, 449-471. doi: 10.1161/CIRCRESAHA.116.308427

Ikram, M. K., De Jong, F. J., Van Dijk, E. J., Prins, N. D., Hofman, A., Breteler, M. M. B., et al. (2006). Retinal vessel diameters and cerebral small vessel disease: the rotterdam scan study. Brain 129, 182-188. doi: 10.1093/brain/awh688

Kawasaki, R., Xie, J., and Cheung, N. (2012 Retinal microvascular signs and risk of stroke: the Multi-Ethnic study of atherosclerosis (MESA). Stroke 43, 3245-3251. doi: 10.1161/STROKEAHA.112.673335

Kim, M., Park, K. H., Kwon, J. W., Jeoung, J. W., Kim, T. W., and Kim, D. M. (2011). Retinal nerve fiber layer defect and cerebral small vessel disease. Invest. Ophthalmol. Vis. Sci. 52, 6882-6886. doi: 10.1167/iovs. $11-7276$

Kunz, A., and Iadecola, C. (2009). Cerebral vascular dysregulation in the ischemic brain. Handb. Clin. Neurol. 92, 283-305. doi: 10.1016/S0072-9752(08) 01914-3

Lernfelt, B., Forsberg, M., Blomstrand, C., Mellström, D., and Volkmann, R. (2002). Cerebral atherosclerosis as predictor of stroke and mortality in representative elderly population. Stroke 33, 224-229. doi: 10.1161/hs0102. 102009

Lim, H. B., Kim, Y. W., Kim, J. M., Jo, Y. J., and Kim, J. Y. (2018). The importance of signal strength in quantitative assessment of retinal vessel density using optical coherence tomography angiography. Sci. Rep. 8:12897. doi: 10.1038/s41598-018-31321-9

Liu, L., Gao, J., Bao, W., Hu, C., Xu, Y., Zhao, B., et al. (2018). Analysis of foveal microvascular abnormalities in diabetic retinopathy using optical coherence tomography angiography with projection artifact removal. J. Ophthalmol. 2018, 1-9. doi: 10.1155/2018/3926745

Liu, J., Zhu, Y., Wu, Y., Liu, Y., Teng, Z., Hao, Y., et al. (2017). Association of carotid atherosclerosis and recurrent cerebral infarction in the Chinese population: a meta-analysis. Neuropsychiatr. Dis. Treat. 13, 527-533. doi: 10.2147/NDT.S124386

London, A., Benhar, I., and Schwartz, M. (2013). The retina as a window to the brain-from eye research to CNS disorders. Nat. Rev. Neurol. 9, 44-53. doi: $10.1038 /$ nrneurol.2012.227
Lyden, P. (2017). Using the National Institutes of health stroke scale. Stroke 48, 513-519. doi: 10.1161/STROKEAHA.116.015434

Mitchell, P., Wang, J. J., Wong, T. Y., Smith, W., Klein, R., and Leeder, S. R. (2005). Retinal microvascular signs and risk of stroke and stroke mortality. Neurology 65, 1005-1009. doi: 10.1212/01.wnl.0000179177. 15900.ca

Moss, H. E. (2015). Retinal vascular changes are a marker for cerebral vascular diseases. Curr. Neurol. Neurosci. Rep. 15:40. doi: 10.1007/s11910-0150561-1

Ong, Y. T., De Silva, D. A., Cheung, C. Y., Chang, H.-M., Chen, C. P., Wong, M. C., et al. (2013). Microvascular structure and network in the retina of patients with ischemic stroke. Stroke 44, 2121-2127. doi: 10.1161/STROKEAHA.113. 001741

Ong, Y. T., Hilal, S., Cheung, C. Y., Venketasubramanian, N., Niessen, W. J., Vrooman, H., et al. (2015). Retinal neurodegeneration on optical coherence tomography and cerebral atrophy. Neurosci. Lett. 584, 12-16. doi: 10.1016/j. neulet.2014.10.010

Osborne, N. N., Casson, R. J., Wood, J. P., Chidlow, G., Graham, M., and Melena, J. (2004). Retinal ischemia: mechanisms of damage and potential therapeutic strategies. Prog. Retin. Eye. Res. 23, 91-147. doi: 10.1016/j.preteyeres.2003. 12.001

Pujari, A., Bhaskaran, K., Sharma, P., Singh, P., Phuljhele, S., Saxena, R. et al. (2020). OCTA in neuro-ophthalmology: current clinical role and future perspectives. Surv. Ophthalmol. doi: 10.1016/j.survophthal.2020.10.009. [Online ahead of print].

Rowe, F. J. (2017). Stroke survivors' views and experiences on impact of visual impairment. Brain Behav. 7:e00778. doi: 10.1002/brb3.778

Smith, E. E., Schneider, J. A., Wardlaw, J. M., and Greenberg, S. M. (2012). Cerebral microinfarcts: the invisible lesions. Lancet Neurol. 11, 272-282. doi: 10.1016/S1474-4422(11)70307-6

Sprodhuber, A., Wolz, J., Budai, A., Laumeier, I., Audebert, H. J., and Michelson, G. (2018). The role of retinal vascular density as a screening tool for ageing and stroke. Ophthalmic. Res. 60, 1-8. doi: 10.1159/ 000488491

Tick, S., Rossant, F., Ghorbel, I., Gaudric, A., Sahel, A. J., Ghorbel, I., et al. (2011). Foveal shape and structure in a normal population. Invest. Ophthalmol. Vis. Sci. 52, 5105-5110. doi: 10.1167/iovs.10-7005

Vermeer, S. E., Longstreth, W. T.Jr., and Koudstaal, P. J. (2007). Silent brain infarcts: a systematic review. Lancet Neurol. 6, 611-619. doi: 10.1016/S14744422(07)70170-9

Wang, J. J., Baker, M. L., Hand, P. J., Hankey, G. J., Lindley, R. I., Rochtchina, E., et al. (2011). Transient ischemic attack and acute ischemic stroke: associations with retinal microvascular signs. Stroke 42, 404-408. doi: 10.1161/STROKEAHA.110.598599

Wylęgała, A. (2018). Principles of OCTA and applications in clinical neurology. Curr. Neurol. Neurosci. 18:96. doi: 10.1007/s11910-018-0911-x

Yang, J., Wang, E., Zhao, X., Xia, S., Yuan, M., Chen, H., et al. (2019). Optical coherence tomography angiography analysis of the choriocapillary layer in treatment-naive diabetic eyes. Graefes. Arch. Clin. Exp. Ophthalmol. 257, 1393-1399. doi: 10.1007/s00417-019-4326-x

Zhou, M., Wang, H., Zeng, X., Yin, P., Zhu, J., Zhu, W., et al. (2019). Mortality, morbidity, and risk factors in China and its provinces, 1990-2017: a systematic analysis for the global burden of disease Study 2017. Lancet 394, 1145-1158. doi: 10.1016/S0140-6736(19)30427-1

Conflict of Interest: The authors declare that the research was conducted in the absence of any commercial or financial relationships that could be construed as a potential conflict of interest.

Copyright (c) 2021 Kwapong, Yan, Hao and Wu. This is an open-access article distributed under the terms of the Creative Commons Attribution License (CC BY). The use, distribution or reproduction in other forums is permitted, provided the original author(s) and the copyright owner(s) are credited and that the original publication in this journal is cited, in accordance with accepted academic practice. No use, distribution or reproduction is permitted which does not comply with these terms. 